\title{
2020 ESC Guidelines \\ on Sports Cardiology \\ and Exercise in Patients \\ with Cardiovascular \\ Disease
}

Anaya Malik

Editorial Assistant

Citation: EMJ Cardiol. 2020;8[1]:23-25.

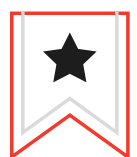

\begin{abstract}
$\Lambda$ DVISING individuals with diseases of the heart on what types and intensities of sport to participate in is not a practice cardiologists typically have official guidelines on. A taskforce from ESC has now come together and created guidelines, the first of their kind, on exercise and sports participation in patients with cardiovascular disease. The guidelines were presented at the ESC Congress 2020 in a session chaired by Prof Antonio Pelliccia, Scientific Director of the Institute of Sports Medicine \& Science from Rome, Italy.
\end{abstract}

Pelliccia was joined by Prof Martin Halle, President of the European Association of Preventive Cardiology (EAPC), Munich, Germany, and Prof Matthias Wilhem, Head of the Centre for Preventive Cardiology, Sports Medicine, Department of Cardiology at the Inselspital, University Hospital of Bern, Switzerland. The guidelines derived from the need to assist patients who had experienced cardiovascular events and were questioning their limits of sports participation. Prof Halle commented on his experience in the taskforce: "The level of evidence is rather low, so it is very much the personal perspective and the experience of the experts which made us come to that one conclusion in the guidelines. It is something that should be developed in years to come."
A series of videos were shown, presented by specialists who were invited to discuss some of the most relevant topics of the guidelines.

\section{EXERCISE AND SPORT FOR RISK MANAGEMENT OF CARDIOVASCULAR DISEASE}

Prof Halle addressed the risk assessment and exercise recommendations for healthy individuals, patients who may be at risk of cardiovascular disease, and the elderly. According to the guidelines, healthy individuals are advised to perform at least 150 minutes of endurance exercise weekly at moderate intensity, or 75 minutes at vigorous intensity, preferably 


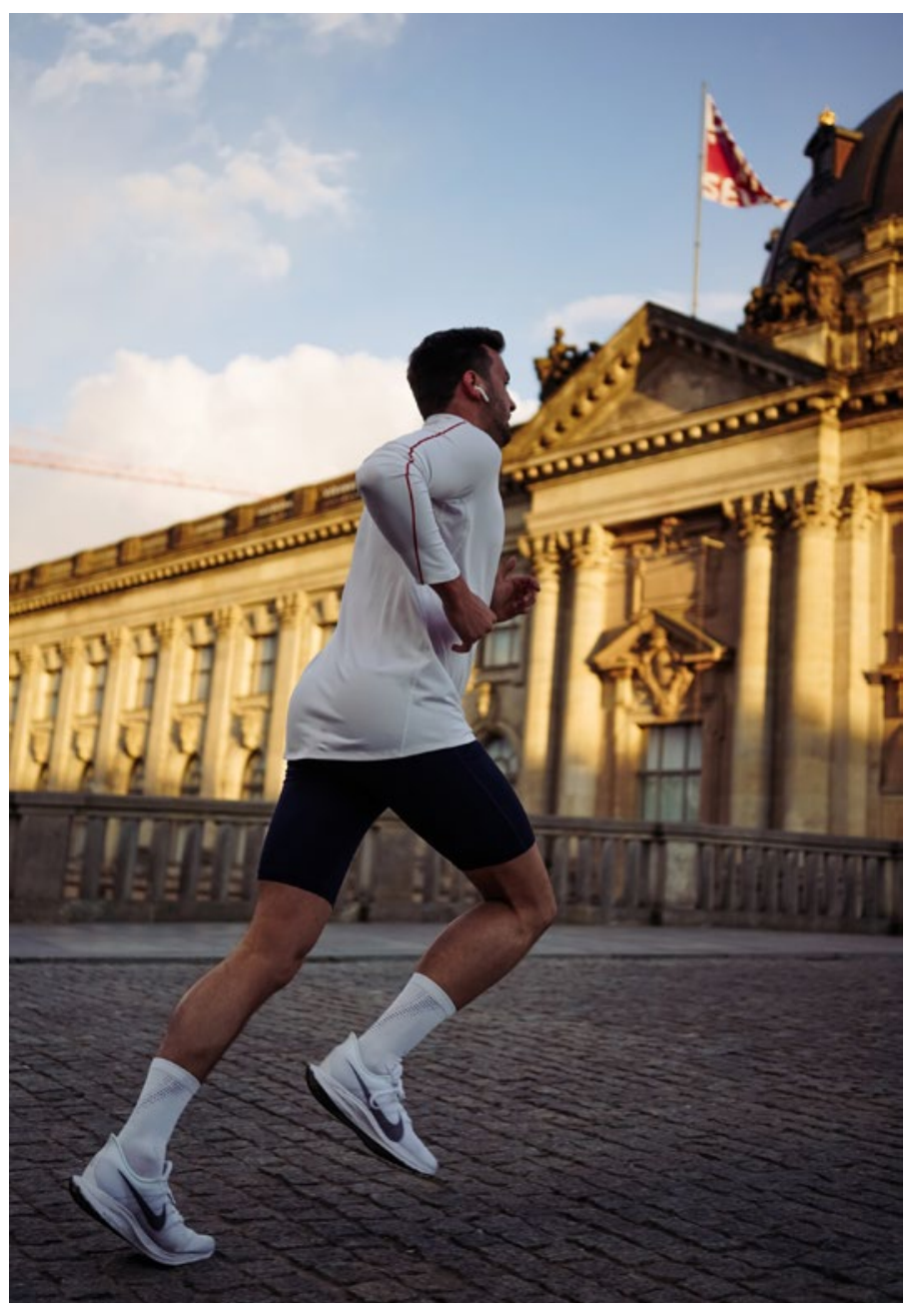

EXERCISE IN VALVULAR HEART DISEASE

The guidelines for exercise and sports recommendations in individuals with valvular heart disease were introduced in the form of short key messages by Dr Sabiha Gati, Royal Brompton and Harefield Hospitals, London, UK:

1. It is recommended that all individuals with valvular heart disease do some form of exercise given the multiple benefits of physical activity.

2. Asymptomatic individuals with mild valvular abnormalities can participate in all recreational and competitive sports. Individuals with severe valvular abnormalities should not participate in intensive exercise.

3. The management of individuals with valvular heart disease requires assessment of their symptomatic status with clinical history, ECG looking for strain patterns, echocardiography with a focus on the valve morphology and function, and exercise stress testing.
4. It is unclear whether exercise accelerates aortic dilation in bicuspid aortic valves in the long term; therefore, a cautious approach to sports participation is recommended.

5. Mitral valve prolapse has a benign nature meaning asymptomatic individuals with mildto-moderate regurgitation can participate in all competitive and recreational sports.

\section{EXERCISE IN PATIENTS AT RISK OF ARRHYTHMIAS AND SUDDEN CARDIAC DEATH}

Prof Hein Heidbuchel, Professor and Chair of Cardiology at Antwerp University, Antwerp, Belgium, was the last expert to present the new guidelines, giving an overview of arrhythmias and channelopathies. He explained that the recommendations in the guidelines for athletes with arrhythmias are based on three main considerations: the risk of sudden cardiac death during sports, sports performance limited by symptoms, and the impact of the sport on arrhythmogenic condition progression. Prof Heidbuchel noted that research and data are limited in this field, therefore shared decision making and discussion with the patient are vital.

\section{CONCLUDING REMARKS}

In the last part of the presentation, Prof Pelliccia led a live question and answer session with questions from experts viewing the discussion from all over the world. Prof Pelliccia then ended the session by inviting his colleagues to read the novel guidelines which contain more topics than those covered in the live session. He shared his ambition to provide a referral document for all cardiologists and examining physicians for patients who ask about exercise, before reflecting on the goals the taskforce set to achieve in creating the guidelines: "We tried to provide the instruments and knowledge for assessing the clinical status of the patient and to give him or her the best advice to approach exercise and sport." As a stimulus for additional research in a field with limited trial data, the comprehensive guidelines presented at ESC for sports cardiology and exercise in patients with cardiovascular disease are sure to aid in closing the research gap. 